\title{
Oscillating gas flow induces reptation of granular droplets
}

\author{
Javier C. Pastenes ${ }^{\dagger}$, Jean-Christophe Géminard ${ }^{\ddagger}$, and Francisco Melo ${ }^{\dagger}$ \\ $\dagger$ Departamento de Física Universidad de Santiago de Chile, \\ Avenida Ecuador 3493, 9170124 Estación Central, Santiago, Chile. \\ $\ddagger$ Laboratoire de Physique, Ecole Normale Supérieure de Lyon, \\ Université de Lyon, CNRS, UMR 5672, 46 Allée d'Italie, F-69007 Lyon, France.
}

(Dated: July 15, 2013)

\begin{abstract}
We report on the reptation of vertically-vibrated droplets of fine particles lying on a solid incline. On the one hand, time-resolved measurements show that the gas pressure in the gap between the droplet bottom and the solid surface can be accounted for by a Darcy law. The cumulative effect of the viscous drag is responsible for the droplet formation. On the other hand, we show that the gap pressure is responsible for an effective horizontal acceleration whose cumulative effect is the upward reptation of the droplets. Using various geometries of the solid substrate, we manipulate the droplets and study the effects of the substrate geometry and of the experimental parameters on the droplet shape and dynamics. The experimental results are discussed in the light of theoretical arguments. This study demonstrates that, by the choice of a suitable geometry of the surface and characteristics of the vibration, one can develop tools for precise powder handling and control.
\end{abstract}

PACS numbers: 45.70.Mg, 45.70.Qj, 81.05.Rm.

\section{INTRODUCTION}

In granular compacts formed of relatively small particles, a rich dynamics arises as the result of the interactions between the grains and the interstitial fluid. Booming sand 1, 2] as well as the granular jet resulting from the impact of a solid object onto the surface of a granular bed [3 5 , both illustrate the complexity of the coupling of a granular system with the interstitial fluid dynamics.

A thick layer of granular material, initially flat and horizontal, subjected to a vertical vibration, exhibits heaping and convection [6 - 9]. The phenomenon is due to the periodic gas flow in the interstices between grains. Heaping and convection have attracted considerable attention and debate, mainly because of the difficulty to discriminate the effects of the solid friction from those of the gaseous drag. To our knowledge, a simple functional relation between the convection intensity and the driving force is still lacking.

When the granular layer is sufficiently thin, under periodic tapping, granular droplets nucleate instead [10. The droplets formation has been attributed to the interplay between the air flow through the material and the avalanche properties, which led to propose a scaling for the droplet size [10]. The granular droplets dynamics was found to share common features with the liquid counterpart, leading to propose a close analogy between the governing equations of the granular droplets and those of partially wetting liquids 10 . Although this analogy was tempting it has not been explored further, likely due to the lack of a precise definition of the surface tension for the granular materials [1].

\footnotetext{
* francisco.melo@usach.cl
}

If the bottom of the container submitted under vertical vibration deviates from the horizontal, a striking effect arises: droplets, instead of moving downhill as could be expected, climb up [12. Moreover, droplets grow longer, like water droplets flowing along a wall, when the inclination angle is increased. Their velocity is observed to be independent of their size but to increase almost linearly with the tilt angle. However, when the angle exceeds the repose angle, the droplets loose stability and form elongated fingers which point upwards; the heads of the fingers quickly shrink by loss of mass while they climb. For inclination angles larger than the avalanche angle, droplets and fingers are no longer observed. In our previous work, the main experimental parameters were explored and a qualitative interpretation of the droplets dynamics was given [12. However, the mechanism proposed to explain such non intuitive behaviors remained to be proven by quantitative comparison with the experimental data.

In the present article, we approach the study of the granular droplets motion from a heuristic point of view. By designing an experimental tool to assess the instantaneous gas pressure at the droplet base, we investigate quantitatively the driving mechanism. We show that droplet formation and reptation results from the cumulative effect of the horizontal component of the viscous drag force due to the gas flow. At relatively low frequency, a Darcy law accounts well for the functional dependence of the droplet velocity on its size and on the inclination angle, vibration frequency and acceleration. In order to control the trajectories, we modify the surface geometry and observe that convex surfaces concentrate the droplets whereas concave ones disperse them. The selection of the principal curvatures thus makes it possible to guide the droplets along trajectories that are the crest lines of the surface. 


\section{EXPERIMENTAL METHOD}

(a)
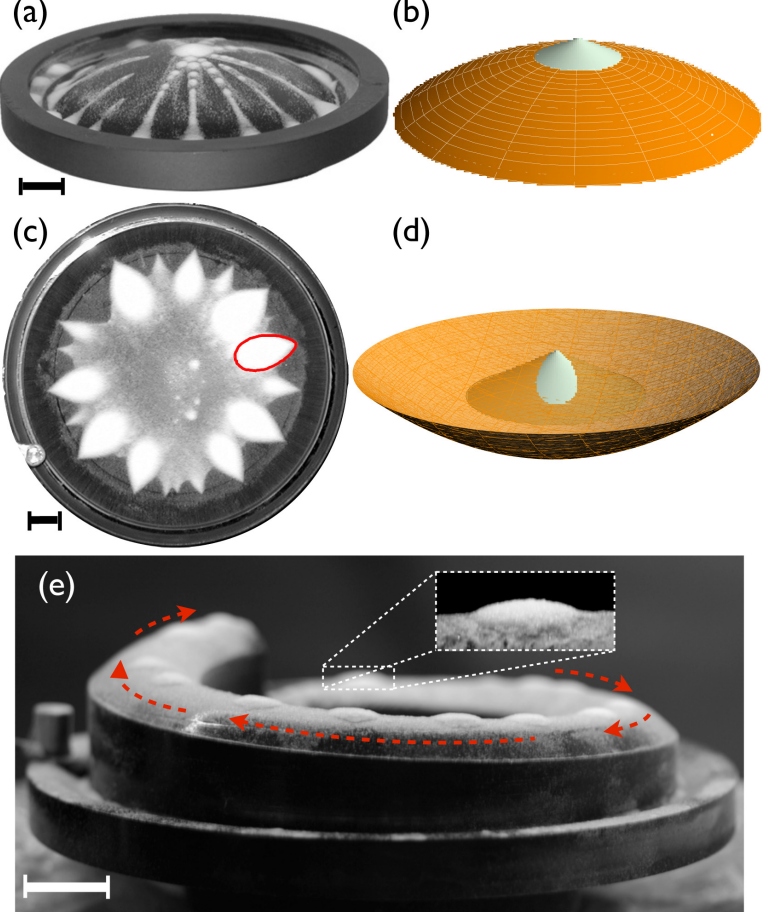

FIG. 1. (Color online) (a) Grazing view of droplets climbing on a convex surface. (c) Top view of petals on a concave surface. (b) and (d) Droplet shapes obtained as the intersects of a vertical cone with the vibrating surface [The line in (c) is a contour obtained with this procedure]. (e) Droplets in a helical cell. Dashed arrows indicate the droplets trajectories. Dashed box: enlargement of a droplet [Scale bars: $10 \mathrm{~mm}$ ].

The droplets dynamics is investigated in various forms of containers which are vertically vibrated using an electrodynamic modal shaker fed with a sinusoidal wave of frequency $f$ ranging from 15 to $60 \mathrm{~Hz}$. The vertical acceleration of the container is monitored by means of an accelerometer with an accuracy of $0.01 \mathrm{~g}$. From the maximum acceleration and from the frequency, one can estimate the dimensionless acceleration $\Gamma=A \omega^{2} / g$ and the maximum amplitude $A$ (We denote $g$ the acceleration due to gravity and $\omega=2 \pi f)$. The granular material consists of silica grains of density $\rho=(0.49 \pm 0.01) \mathrm{gr} / \mathrm{cc}$ and diameter ranging from $d=(50 \pm 15) \mu \mathrm{m}$ to $d=(200 \pm 40)$ $\mu \mathrm{m}$, excepted when specified. Layer depths range from $2.5 \mathrm{~mm}$ to $10 \mathrm{~mm}$. A high-speed video camera provides either top or lateral views of the droplets with a spatial resolution of $256 \times 256 \mathrm{px}^{2}$ at 1200 frames per seconds.

From the previous experimental studies, we know that the droplets diffuse across a flat surface and that, as a consequence of their upwards motion, they tend to reach the top of convex surfaces [12, as shown in figure 1 (a); since the droplets motion proceeds along the local maximum slope, they reach the minimum slope. We take ad-

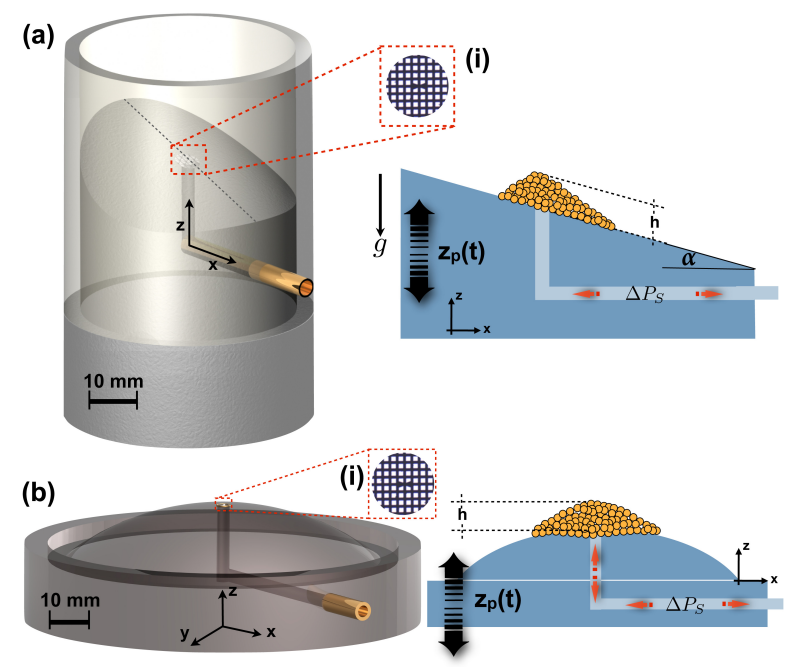

FIG. 2. (Color online) (a) Inclined bottom cell with view of the grid (i) protecting the cavity. Right panel: Sketch of a moving droplet passing above the grid. (b) Convex cell. Right panel: Sketch of the stationary droplet at the top of the convex surface.

vantage of this effect to accurately measure the pressure at the bottom of either a moving or a steady granular droplet.

In a first experimental configuration, in order to measure the gas pressure, $P(t)$, at the base of a droplet in motion and, thus, to elucidate the driving mechanism, a single curvature cell was designed. The cell is made of a plexiglass tube $(46 \mathrm{~mm}$ inner diameter and $80 \mathrm{~mm}$ height) glued to an aluminum mount. The bottom is a cylinder whose axis makes the angle $\alpha$ with the horizontal [Fig. 2(a)]. In this geometry, the droplets climb up following the rectilinear crest line of the surface. The measure of the pressure is made possible by an internal L-shaped cavity (2 $\mathrm{mm}$ in radius) drilled in the aluminum mount. At one end, the cavity emerges somewhere along the crest line and thus intersects the droplets trajectory. A flush-mounted grid (mesh $45 \mu \mathrm{m}$ ) avoids that the particles enter the cavity. The other end is connected to a differential pressure sensor (in the range $-125 \mathrm{~Pa}$ to 125 $\mathrm{Pa}$ ) through a hose. The use of a non-torsional hose prevents undesired pressure variations likely due to its own deformation.

In a second experimental configuration, in order to systematically study the dependence of the excess pressure at the base of the droplet on the experimental parameters, we use a convex cell (97 $\mathrm{mm}$ in diameter), as depicted in Fig. 2(b). The pressure underneath the granular droplet is measured as described above; the cavity arises exactly at the top of the surface (radius of curvature $57.5 \mathrm{~mm}$, much larger than the typical droplet size). The droplet remains steady and the gap pressure is periodic. 

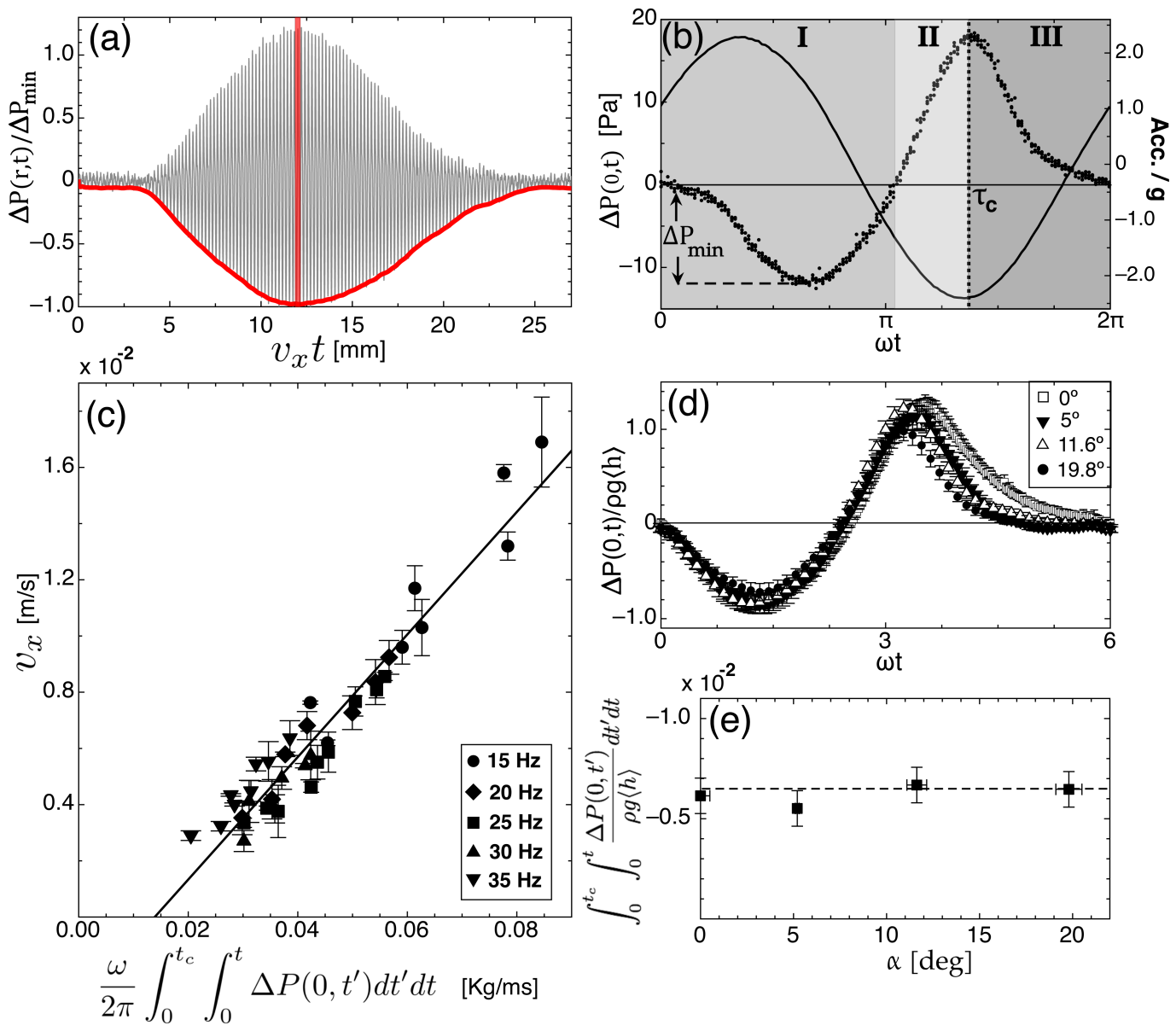

FIG. 3. (Color online) (a)Pressure signal associated with a droplet climbing at $5.8 \mathrm{~mm} / \mathrm{s}[$ silica particles $d=100 \mu \mathrm{m}, f=30 \mathrm{~Hz}$, $\Gamma=3.0$, and $\alpha=12.8^{\circ}$. (b) Details of pressure cycle close to the maximum of the pressure variations - I Depression; right after droplet taking off. II Overpressure; during droplet return to the plate. III Pressure relaxation; after the droplet landing on the plate. The container acceleration is indicated by the solid line. (c) Droplets velocity as a function of the integrated pressure [Eq. (1)] for distinct $f$; integration limits $\left[0, \tau_{c}=\omega t_{c}\right]$ are defined in (b). (d) Pressure cycles for various surface inclination, $\alpha$, showing superposition for $d=100 \mu \mathrm{m}, f=20 \mathrm{~Hz}, \Gamma=2.2$. (e) Integrated pressure as function of $\alpha$.

\section{EXPERIMENTAL RESULTS}

\section{A. Droplet shape}

Basically, the droplet formation originates from the migration of the surface grains toward the hills under the action of the gas flow. The granular convection is not significant, and after a few collisions with the plate the droplets acquire stationary conical shapes, although the largest droplets (on flat and convex surfaces) have tendency to be rounded at the top [Fig. 11(a)]. On the convex surface, the droplets grow longer along the maximum slope. On the concave surface [Fig. 11(c)], the droplets are petal-shaped. These shapes which are apparently very different are accounted for by considering the intersect of the vibrating surface and of a vertical cone exhibiting the same slopes as the droplet [Fig. 1(d)]. The apex of the cone coincides with the droplet apex. This simple geometrical construction is in good agreement with the experimental shapes [Fig. 1(c)].

\section{B. Droplet velocity and driving mechanism}

The first experimental configuration makes possible to measure the pressure difference, $\Delta P(t)=P(t)-P_{0}$ ( $P_{0}$ stands for the ambient pressure), during the whole droplet transit above the grid [Fig. 3(a)].

Within a cycle, $\Delta P(t)$ has three stages as depicted in Fig. 3(b): a depression (zone I) beginning immediately after the droplet take-off (occurring when the container acceleration equals $-g$ ), an overpressure (zone II) observed as the droplet approaches the vibrating surface, and a pressure relaxation (zone III) due to the gas evacuation through the droplet which enters in contact with the surface at $t_{c}$, compacts and finally comes to rest [13]. The envelope of such signal, given that the droplet velocity is known, provides an indication of the pressure profile along the droplet bottom. The amplitude of the 
pressure variations is maximum when the droplet apex is at the vertical of the grid.

The mechanism driving the droplet motion is now seen as follows. During the take-off, the pressure in the gap between the droplet base and the solid surface decreases, which leads to a net force, normal to the surface, pushing the droplet toward the surface. Integration of the pressure profile across the droplet base provides us with an estimate of the intensity of the latter force and, thus, of its horizontal component. One can then write the equation governing the motion of the droplet center of mass along the horizontal. Considering a droplet of average height $\langle h\rangle$ and volume $V=S\langle h\rangle$, climbing along a slope making the angle $\alpha$ with the horizontal, one gets $\rho V \frac{d^{2} x(t)}{d t^{2}}=\int_{S}\left(P(\vec{r}, t)-P_{0}\right) \sin (\alpha) d S$, where $x(t)$ is the horizontal position of the droplet, $P(\vec{r}, t)$ the pressure at the position $\vec{r}$ at time $t$ in the plane of the droplet base, and $\mathrm{S}$ the surface area of the droplet bottom. The average horizontal velocity of the droplet can then be estimated under the form

$$
v_{x}=\frac{\omega}{2 \pi} \frac{\sin (\alpha)}{\rho\langle h\rangle}\left[\int_{0}^{t_{c}} \int_{0}^{t}\left\langle\Delta P\left(t^{\prime}\right)\right\rangle_{S} d t^{\prime} d t\right]
$$

where $\langle\Delta P(t)\rangle_{S}$ is the average, over the surface $\mathrm{S}$, of the pressure difference $P(\vec{r}, t)-P_{0}$ at time t.

In order to check Eq. (1) experimentally, we produced droplets having the same average height, $\langle h\rangle$, by ensuring that all the material in the cell was forming a single droplet. We measured their velocity and the associated pressure variations. In order to estimate the effects of the driving force, we consider, instead of $\langle\Delta P(t)\rangle_{S}$, the pressure difference $\Delta P(t)$ during the pressure cycle having the maximum amplitude [Fig. 3(b)]. One can indeed guess that $\langle\Delta P(t)\rangle_{S}=C \Delta P(t)$ with $C$ a geometrical factor smaller than unity. The data displayed in Fig. 3(c) corroborate the linear dependence of the droplet velocity on the integrated effect of the driving force for distinct driving frequencies. The experimental slope leads to $C \approx 0.85$, which indicates that the pressure varies slowly across the droplet base, except near the periphery where some gas leak might be present. In our previous work [12] we showed that $v_{x}$ is a nearly linear function of $\alpha$, which is consistent with Eq. (1) if the pressure variation is independent of $\alpha$. Consistently, Fig. 3(d) displays raw pressure data indicating that the pressure cycle remains almost unchanged when the angle $\alpha$ is varied. Moreover, Fig. 3(e) shows that the integrated pressure is indeed nearly independent on $\alpha$, supporting the validity of Eq. (1). It is worth noting that the size of the grid limits the smallest particle size that can be characterized to $d>45 \mu \mathrm{m}$. This fact, along with the observation that droplets are no longer robust for $d>200 \mu \mathrm{m}$, makes it difficult to determine the dependence of $v_{x}$ on the diameter $d$ of the particles. Despite this difficulty, we observed that at fixed $\Gamma$ and $f, v_{x}$ exhibits little dependence on $d$.

\section{Pressure pattern vs. experimental parameters}

In order to systematically study the dependence of pressure pattern $\Delta P(t)$ on the experimental parameters, we use the second experimental configuration. We thus consider the periodic variations of the pressure underneath the droplet bouncing vertically at the top of the convex surface. As before, three stages are distinguished in a pressure cycle [Fig. 4(a)]

We propose a simplified approach to account for the experimental pattern. Indeed, the precise calculation of the temporal evolution of the gap pressure would require to find the self-consistent droplet shape, which is difficult. Thus, in a first simplified approach, we neglect the grain convection and, even, consider that the droplet does not deform and exhibits a simple conical shape. On the one hand, the droplet is submitted to the pressure force so that the altitude, $z(t)$, of the droplet center of mass in the frame of reference moving with the solid surface obeys $\ddot{z}(t)+\ddot{z}_{p}(t)=-g+\frac{1}{\rho V} \int_{S_{T}} P(r, t) \hat{\mathbf{z}} \cdot \mathbf{d} \mathbf{S}$, where $z_{p}(t)=A \sin \omega t$ is the vertical position of the solid surface and $\hat{\mathbf{z}}$ the vertical unit vector. The integral is calculated over the whole area, $S_{T}$, of the external surface of the droplet. Considering the average pressure $\langle\Delta P(t)\rangle_{S}$ at the droplet bottom at time $t$, one can rewrite the latter relation in the form $\ddot{z}(t)+\ddot{z}_{p}(t)=\frac{S}{\rho V}\langle\Delta P(t)\rangle_{S}-g$. On the other hand, the pressure difference across the droplet induces a gas flow through the grains. The instantaneous flow-rate is approximately given by a Darcy law, $\mathbf{q}=-\frac{\kappa}{\eta} \nabla P$, where $\eta$ is the gas viscosity. The permeability $\kappa$ is given by the Ergun relation, $\kappa=\frac{\psi^{3} d^{2}}{150(1-\psi)^{2}}$, where $\psi$ is the porosity [14. Finally, assuming that the gas is incompressible [15, one can estimate that the variation $\dot{s}(t)$ of the gap $s(t)$ between the droplet base and the solid surface is only permitted by the gas flow through the droplet, which imposes that $\dot{s}=q_{z}$, with $q_{z}=\frac{\kappa}{\eta} \frac{\langle\Delta P(t)\rangle_{S}}{\langle h\rangle}$. In addition, notice that in absence of dilation of the droplet, $\dot{z}=\dot{s}$. Thus, combining the equation governing the vertical motion of the droplet and the condition imposed by the gas incompressibility, one gets

$$
\ddot{s}(\tau)+\frac{\dot{s}(\tau)}{\tau_{\kappa}}=\frac{A}{\Gamma}\left[\Gamma \sin \left(\tau+\tau_{0}\right)-1\right],
$$

where we defined $\tau=\omega t$ and $\tau_{0}=\omega t_{0}=\arcsin 1 / \Gamma$, $t_{0}$ being the time of the droplet take-off. The parameter $\tau_{\kappa}=\omega \kappa \rho / \eta$ is a relaxation time. Eq. (2) was first obtained for a porous oscillating piston (the Kroll's model) and has an analytic solution which writes [16],

$$
\begin{aligned}
\langle\Delta P(\tau)\rangle_{S} & =-\frac{\rho g\langle h\rangle}{1+\tau_{\kappa}^{2}}\left[\sqrt{\Gamma^{2}-1}\left(\sin \tau-\tau_{\kappa} \cos \tau\right)\right. \\
& +\tau_{\kappa} \sin \tau-\tau_{\kappa}^{2}+\cos \tau \\
& \left.+\tau_{\kappa}\left(\sqrt{\Gamma^{2}-1}+\tau_{\kappa}\right) e^{-\frac{\tau}{\tau_{\kappa}}}-1\right] .
\end{aligned}
$$

The pressure difference exhibits a minimum, $\langle\Delta P\rangle_{S, \text { min }}$, which, in the limit of small $\tau_{\kappa}(<0.7)$ 

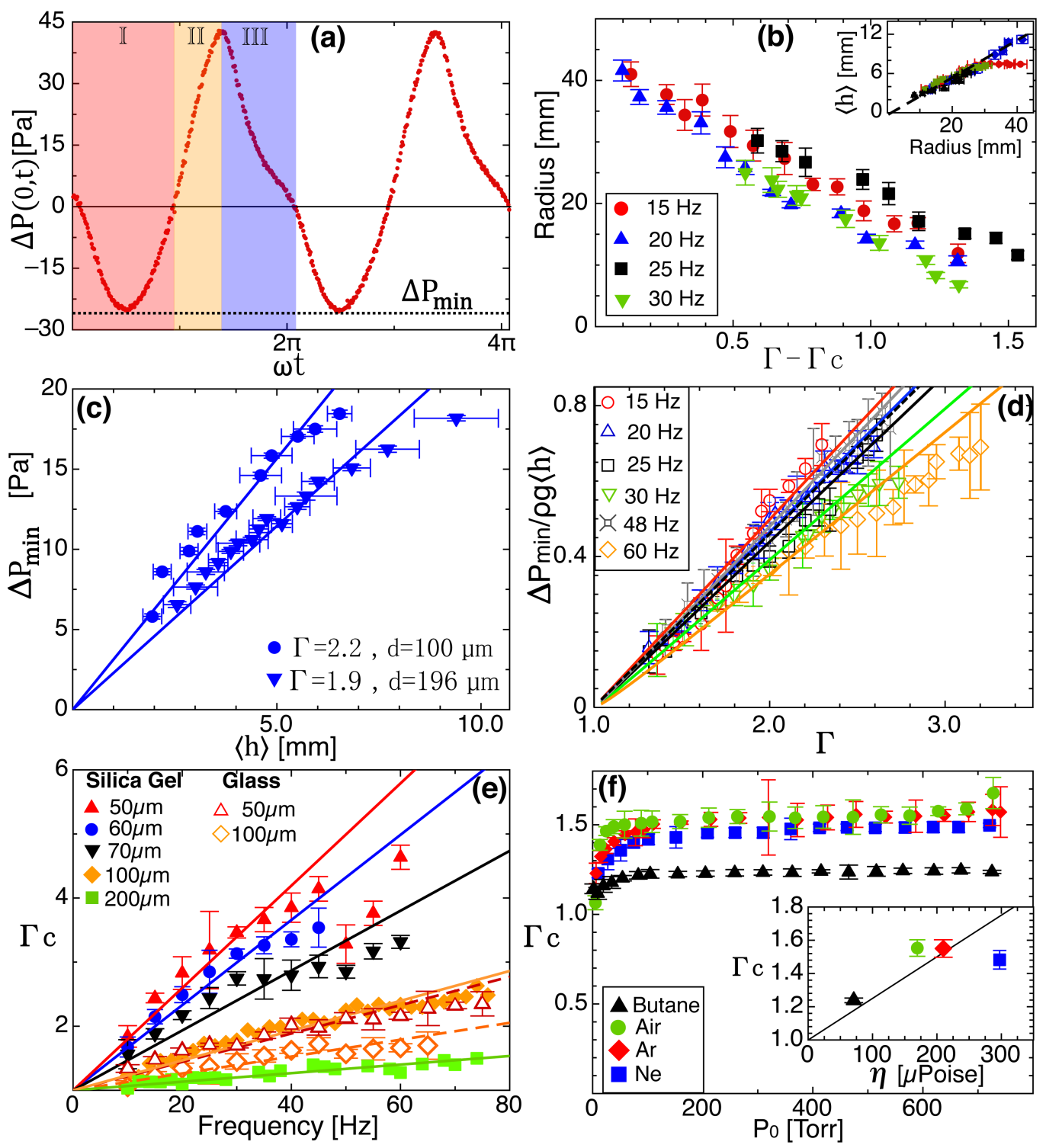

FIG. 4. (Color online) (a) Typical gap pressure showing three main stages I Depression; right after droplet taking off. II Overpressure; during droplet return to the plate. III Pressure relaxation $[\Gamma=2.6, f=15 \mathrm{~Hz}]$. (b) Maximum droplet radius as function of $\Gamma$ for distinct $f$. Inset, droplet height vs droplet radius; proportionality is in agreement with the (supposed) conical shape. (c) Maximum depression versus droplet height $[f=20 \mathrm{~Hz}]$. (d) Maximum depression vs $\Gamma$ for distinct $f$. Straight lines are the best adjustment to the Eq. (4) which leads to $\psi \approx 0.54$. [(a)-(d) Silica gel, $d=100 \mu \mathrm{m}$. (e) Creep onset, $\Gamma_{c}$, vs $f$ for distinct $d$, silica gel and glass particles. Solid lines are best fits to Eq. (6) with $\beta=0.3$. (f) $\Gamma_{c}$ for variable gas pressure $P_{0}$ for distinct gases [silica gel, $d=100 \mu \mathrm{m}, f=20 \mathrm{~Hz}$ ]. Inset, $\Gamma_{c}$ vs $\eta$ for increasing $\eta$ : Butane, Air, Argon and Neon. Solid line is from Eq. (6) with $\beta=0.3$.

and $\Gamma$ slightly larger than 1 , is obtained by minimization of Eq. (3) with respect to $\tau$, leading to:

$$
\langle\Delta P\rangle_{S, \text { min }} \approx-\rho g\langle h\rangle\left[(\Gamma-1)-\frac{\Gamma}{2} \tau_{\kappa}^{2}\right] .
$$

Assuming that the pressure difference measured at the center of the droplet base $\Delta P(0, \tau)$ [Fig. 4(a)] differs from $\langle\Delta P(\tau)\rangle_{S}$ only by a multiplicative factor, one estimates that the maximum amplitude of the pressure variations is simply proportional to $\langle\Delta P\rangle_{S, \min }$. With this assumption, Eq. (4) correctly predicts the amplitude of the pressure variations is proportional to both $\langle h\rangle$ and $(\Gamma-1)$, in agreement with the experimental data displayed in Fig 4(c) and (d) respectively. We mention that $\langle\Delta P\rangle_{S, \text { min }}$ is a slowly varying function of $\tau_{\kappa}$ and that the regime for large $\tau_{\kappa}>>1$ is neither captured by Eq. (4) nor observed for the parameter range explored in the experiments. 


\section{DISCUSSION}

In the present section, we discuss thoroughly the consequences of the relations established above.

First, we consider the condition for the droplet formation. A condition for the vibration to heap the grains is that the work of the pressure in a cycle must exceed the potential energy necessary to rise a single layer of grains over a height equal to the layer thickness. The condition can be written $\int\left(\langle P\rangle_{S}-P_{0}\right) d z \geq \rho g\langle h\rangle d$, which leads to the minimum dimensionless acceleration, $\Gamma_{h}$, given by $\left(\Gamma_{h}-1\right) \Gamma_{h} \geq \frac{d \omega^{2}}{g}$ in the limit $\tau_{\kappa} \ll 1$.

In addition, as shown in Fig. 4(e), the critical acceleration $\Gamma_{c}$ for the droplets to creep is nearly linear with $\omega$ and decreases with $d$. Moreover, $\Gamma_{c}$ depends linearly on the gas viscosity [see inset in Fig. 4(f)] and decreases with increasing material density. Interestingly, the measurements of $\Gamma_{c}$ as a function of the gas pressure show that $\Gamma_{c}$ is not a function of the gas density, which indicates that the relevant parameter is indeed the dynamical viscosity $\eta$ [Fig. 4(f)]. The correct scaling for $\Gamma_{c}$ can be recovered by assuming that a minimum gap, of the order of $d$, is required for the droplet to creep (the droplet effectively takes off, the pertinent scale being given by the grain size). From Eq. (2), by replacing the maximum gap by $\beta d$, with $\beta$ a numeric constant, we find:

$$
\frac{150 \beta(1-\psi)^{2} \eta \omega}{d \psi^{3} g \rho} \approx \int_{0}^{\tau_{s}}\left[\Gamma_{c} \sin \left(\tau+\tau_{0}\right)-1\right] d \tau
$$

where $\tau_{s}$ is the dimensionless time at which the gap is maximum. Interestingly, for $\tau_{\kappa}<<1$, the right-handside of Eq. (5) can be approximated to $\Gamma_{c}-1$, leading to:

$$
\Gamma_{c}-1 \approx \frac{150 \beta(1-\psi)^{2} \eta \omega}{d \psi^{3} g \rho}
$$

Eq. (6) provides a functional dependence on the parameters that is consistent with our experimental data (including those in [12]) as shown in Fig. 4(e). All experimental curves in Fig. 4(e) are fitted with the Eq. (6) by using a single parameter $\beta=0.3$. For $\tau_{\kappa}>1$, the right-handside of Eq. (5) is, in addition, a function of $\tau_{\kappa}$ leading to an implicit expression for $\Gamma_{c}$ that can be calculated numerically.

Finally note that, since the pressure variation is proportional to $\langle h\rangle[\mathrm{Eq}$. (4)], Eq. (1) immediately predicts that the droplet velocity is independent of the droplet size, as confirmed experimentally [12].

\section{CONCLUSIONS}

In summary, we showed that droplets forming and reptation are quantitatively accounted for by measuring and modeling the pressure excess due to the gas flow through the porous granulate. This agreement suggests that gas leak expected to occur at the droplet periphery is not dominant in the range of the parameters we explored experimentally. A simplified model accounts satisfactorily for the dependence of the droplet velocity on the main experimental parameters. For instance, given that the gap pressure is almost independent of $\tau_{\kappa}=\omega \kappa \rho / \eta$ in the limit $\tau_{\kappa}<<1$, the droplet velocity is independent of the gas dynamical viscosity.

In addition, we show that the droplet size can be controlled on a convex surface by selecting the vibration parameters as shown in Fig. 4(b) where the size is decreased by increasing the acceleration in a range of driving frequencies. In turn, droplets trajectories can be defined by the choice of the surface geometry as illustrated in Fig. 1(e), where droplets follow the helical trajectory defined by the crest line of the surface. Thus, combining concave with convex geometries and selecting suitable vibration parameters, a variety of strategies and devices can be devised for powder handling and control (ranging from micro-delivery to separation, [17]).

\section{ACKNOWLEDGMENTS}

The authors acknowledge the financial support from the contracts ANR-09-BLAN-0389-01/Fondecyt-011 and anillo ACT95.
[1] R. A. Bagnold, The Physics of Blown Sand and Desert Dunes. (Methuen, London, 1954).

[2] B. Andreotti, L. Bonneau, and E. Clément, Geophys. Res. Lett., 35, L08306, (2008).

[3] S. T. Thoroddsen and A. Q. Shen, Phys. Fluids, 13, 4, (2001).

[4] D. Lohse, R. Rauhe, R. Bergmann and D. Van Der Meer, Nature, 432, 689, (2004).

[5] S. Deboeuf, P. Gondret and M. Rabaud, Phys. Rev. E, 79, 041306, (2009).

[6] P. Evesque and J. Rajchenbach, Phys. Rev. Lett. 62, 44,
(1989).

[7] C. Laroche, S. Douady and S. Fauve, J. Phys. (Paris), 50, 699, (1989).

[8] H. K. Pak and R. P. Behringer, Phys. Rev. Lett. 71, 1832, (1993).

[9] H. K. Pak, E. Van Doorn, and R. P. Behringer, Phys. Rev. Lett. 74, 4643, (1995).

[10] J. Duran, Phys. Rev. Lett. 87, 4301, (2001).

[11] Y. Amarouchene, J-F. Boudet and H. Kellay. Phys. Rev. Lett. 100, 218001, (2008).

[12] L. Caballero and F. Melo, Phys. Rev. Lett. 93, 258001, 
(2004).

[13] Q. Wu, Y. Andreopoulus, and S. Weinbaum, Phys. Rev. Lett. 93, 19, 4501, (2004).

[14] See for instance, R.M. Nedderman, Statics and Kinematics of Granular Materials, (Cambridge Univ. Press 1992).

[15] Notice that compressibility effect can be neglected when $f \tau_{D}<<1$, with $\tau_{D}=\langle h\rangle^{2} / D$. Where $D \equiv \kappa / \psi \eta \chi$ is the relevant diffusion coefficient and $\chi$ is the gas compressibility. In our case, in air, this effect become significant at relatively low frequencies which are above $60 \mathrm{~Hz}$.

[16] L.I. Reyes, I. Sánchez, G. Gutiérrez, Physica A. 358, 466, (2005).

[17] See for instance, F. J. Muzzio, T. Shinbrot and B. J. Glasser, Powder Technology 124, 1, (2002). 